\title{
Evaluating ESOP Optimization Methods in Quantum Compilation Flows
}

\author{
Giulia Meuli ${ }^{1}$, Bruno Schmitt ${ }^{1}$, Rüdiger Ehlers ${ }^{2}$, \\ Heinz Riener ${ }^{1}$, and Giovanni De Micheli ${ }^{1}$ \\ ${ }^{1}$ École Polytechnique Fédérale de Lausanne, Switzerland \\ ${ }^{2}$ University of Bremen, Germany
}

\begin{abstract}
Exclusive-or sum-of-products (ESOP) expressions are used as intermediate representations in quantum circuit synthesis flows, and their complexity impacts the number of gates of the resulting circuits. Many state-of-the-art techniques focus on minimizing the number of product terms in a ESOP expression, either exactly or in a heuristic fashion.

In this paper, we investigate into ESOP optimization considering two recent quantum compilation flows with opposite requirements. The first flow generates Boolean functions with a small number of Boolean variables, which enables the usage of methods from exact synthesis; the second flow generates Boolean functions with many Boolean variables, such that heuristics are more effective. We focus on the reduction of the number of $T$ gates, which are expensive in fault-tolerant quantum computing and integrate ESOP optimization methods into both flows. We show an average reductions of $36.32 \%$ in $T$-count for the first flow, while in the second flow an average reduction of $28.23 \%$ is achieved.
\end{abstract}

Keywords: reversible logic synthesis · logic optimization - ESOP · quantum circuit

\section{Introduction}

Quantum compilation is the problem of translating a computational description of a quantum algorithm into basic quantum operations. Two main approaches are used in practice: 1) manual compilation, where a designer manually synthesizes (and optimizes) each component of the computational description and generates the final quantum circuit by hand, and 2) automatic compilation, which supports designers in the synthesis task by offering fast and scalable solutions to systematically explore the design space. On the one hand, automatic synthesis allows designers to deal with larger problems that are too complex to be tackled manually; on the other hand, systematic design space exploration enables designers to identify optimization capabilities otherwise overlooked.

Recent attempts in the field of automatic quantum compilation include $L U T$ based Hierarchical Reversible Logic Synthesis (LHRS) 25] and Decomposition Based Synthesis $(D B S)$ [23. The former framework, LHRS, uses a hierarchical 
method to synthesize quantum circuits from specifications provided in form of combinational logic designs. The designs are first decomposed into networks of look-up tables (LUTs). Then, a quantum circuit is assembled by translating each LUT into quantum gates. The latter framework, $D B S$, uses Youngsubgroup based reversible synthesis $[3$ to compile quantum state permutations into quantum circuit. Both frameworks, LHRS and DBS, use exclusive-or sumof-products (ESOPs) as representations of reversible logic gates generated during the translation process.

ESOPs are a classical two-level logic representation consisting of one level of AND-gates, followed by one level of XOR-gates. They provide a compact logic representation of Boolean functions, and are, for some classes of function$\mathrm{s}$, exponentially more compact when compared to the sum-of-products (SOP) representation 21. This compactness can be particularly recognized when XORintensive circuits, such as the parity function, need to be represented and makes ESOPs useful to describe arithmetic and cryptographic primitives [15.

Over the years, many advanced synthesis and optimization methods have been discovered for ESOPs. Exact methods [16, 19,20 target the minimization of the number of product terms in an ESOP, such that the number becomes provably minimal. Their applicability, however, is limited to Boolean functions with at most 7 Boolean variables. Moreover, they often require large tables of precomputed information and need a substantial amount of runtime to guarantee minimality.

Heuristic methods 13,20,27 are capable of reducing large-scale ESOPs with thousands of cubes by repeatedly applying simple cube transformation rules that first expand and then collapse cubes. Such transformation-based optimization strategies are fast, lead to significant reductions, and can be applied even if ESOPs with many Boolean variables are considered. Heuristic methods, however, cannot guarantee optimality and their progress often strongly degrades over time - the chances of finding a pair of cubes that can be collapsed decreases and the improvement saturates.

Overall, in this work, we target fault-tolerant quantum computation and analyze the impact of ESOP optimization methods on the number of $T$ gates of the final quantum circuit. The $T$ gates have been recognized as the most expensive gates in fault-tolerant quantum computing [1].

We integrate advanced ESOP optimization methods, both heuristic and exact, into recent quantum compilation flows. In particular, we consider $L H R S$ and $D B S$ as two possible application scenarios with opposite requirements: $D B S$ uses simple specifications, such that only a few Boolean functions with a relatively small number of Boolean variables have to be synthesized. In this case, exact synthesis methods are useful and allow us to generate ESOPs of provably minimal size. In $L H R S$, however, ESOP optimization has to deal with many and larger Boolean functions. In this case, we advocate heuristic ESOP optimization methods to keep the approach scalable.

In our analysis, we consider two de-facto standard cost functions from logic synthesis - the number of product terms and the number of literals - and propose 
a novel exact synthesis procedure for ESOPs. Our procedure allows users to specify costs for each cube, considered during the synthesis process. We formulate the synthesis problem by introducing a weighted-version of the Helliwell equation 17, and solve the problem using partial weighted MAX-SAT.

\section{Preliminaries}

\subsection{ESOP representation of Boolean functions}

Definition 1. An ESOP over $n$ Boolean variables, $x_{1}, \ldots, x_{n} \in \mathbb{B}$, is an expression of form $t_{1} \oplus \cdots \oplus t_{k}$, where each $t_{i}=l_{i, 1} \cdots l_{i, l_{i}}$ is a product term (or cube) of literals $l_{i, j} \in\left\{x_{1}, \ldots, x_{n}, \bar{x}_{1}, \ldots, \bar{x}_{n}\right\}$ for $1 \leq i \leq k$ and $1 \leq j \leq l_{i}$. The symbol $\oplus$ denotes the modulo-2 addition (XOR-operation), and $\bar{x}_{i}$ denotes the negated Boolean variable $x_{i}$ for $1 \leq i \leq n$.

An ESOP expression can be interpreted as a two-level logic circuit, which realizes a possibly incompletely-specified Boolean function $f:\{0,1,-\}^{n} \rightarrow \mathbb{B}$, i.e., $f\left(x_{1}, \ldots, x_{n}\right)=t_{1} \oplus \cdots \oplus t_{k}$ for all possible valuations of the Boolean variables $x_{1}, \ldots, x_{n}$.

\subsection{ESOP-based reversible logic synthesis}

Reversible circuits are logic networks with the same number of inputs and outputs, composed of reversible gates. The most commonly used gates are the single-target gates and the multiple-controlled Toffoli gates.

Definition 2. Let $c: \mathbb{B}^{k} \rightarrow \mathbb{B}$ be a Boolean function, called control function. Also, let $C=\left\{x_{1}, \ldots, x_{k}\right\}$ be the control lines and let $x_{t} \notin C$ be a target line. Then the single-target gate $T_{c}(C, t): \mathbb{B}^{n} \rightarrow \mathbb{B}^{n}$ is a reversible Boolean function which maps:

$$
\left(x_{1}, \ldots, x_{n}\right) \rightarrow \begin{cases}x_{i} & \text { if } i \neq t \\ x_{t} \oplus c\left(x_{1}, \ldots, x_{k}\right) & \text { otherwise }\end{cases}
$$

Definition 3. If the control function c can be expressed as a single product term $c=\bigwedge_{i=1}^{k}\left(x_{i} \oplus p_{i}\right)$ using a single-target gate $T_{c}(C, t)$, where $p_{i}, 1 \leq i \leq k$, are the polarities of the controls, then we call the gate a multiple-controlled Toffoli gate.

A multiple-controlled Toffoli gate is a reversible gate acting on the bits in $x_{1}, \ldots, x_{k}, x_{t}$, such that the bits in $C$ remain unchanged and the bit $x_{t}$ flips if the control function $c\left(x_{1}, \ldots, x_{k}\right)$ evaluates to true.

ESOP-based reversible synthesis methods are based on the observation that an ESOP can be directly translated into a reversible circuit, as each term of the expression corresponds to a multiple-controlled Toffoli gate [5,6. The method generates as many Toffoli gates as cubes in the expression, all cascaded and targeting the same bit. 


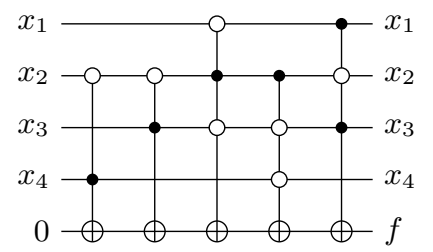

Fig. 1. Example of a reversible circuit of mixed-polarity multiple-controlled Toffoli gates

graph $\longrightarrow \begin{gathered}k \text {-LUT } \\ \text { mapping }\end{gathered} \longrightarrow \begin{gathered}k \text {-LUT } \\ \text { network }\end{gathered} \rightarrow$ pebbling $\longrightarrow \begin{gathered}\text { STG } \\ \text { network }\end{gathered} \rightarrow \begin{gathered}\text { ESOP-based } \\ \text { synthesis }\end{gathered} \longrightarrow \begin{gathered}\text { Toffoli } \\ \text { network }\end{gathered}$

(a) LHRS flow with direct mapping of single-target gates

permutation $\longrightarrow \begin{gathered}\text { Young-subgroup } \\ \text { based synthesis }\end{gathered} \longrightarrow \begin{gathered}\text { STG } \\ \text { network }\end{gathered} \longrightarrow \begin{gathered}\text { ESOP-based } \\ \text { synthesis }\end{gathered} \longrightarrow \begin{gathered}\text { Toffoli } \\ \text { network }\end{gathered}$

(b) $D B S$ flow

Fig. 2. Two different state-of-the-art compilation flows for Boolean functions that use ESOP-based reversible synthesis

Example 1. The Toffoli network in Fig. 1 corresponds to the ESOP expression:

$$
x_{4} \bar{x}_{2} \oplus x_{3} \bar{x}_{2} \oplus \bar{x}_{3} x_{2} \bar{x}_{1} \oplus \bar{x}_{4} \bar{x}_{3} x_{2} \oplus x_{3} \bar{x}_{2} x_{1}
$$

Some optimization techniques aiming at reducing the cost of the generated reversible circuits have been proposed in literature [12, 28]. The final circuit reflects the quality of the ESOP expression, so the synthesis process is crucial for this application.

\section{Optimal ESOP for quantum compilation}

The problem of automatically compiling a Boolean function into a universal quantum library is largely addressed in literature $7,9,22$.

Among the available synthesis methods, hierarchical flows have the capability of being scalable, as they are based on a logic network representation 18, e.g., LHRS [26]. The input to LHRS is a classical logic network, e.g., provided in a hardware description language; the output is a quantum network realized in terms of Clifford $+T$ gates. The framework is based on the usage of $k$-feasible Boolean logic networks ( $k$-LUT networks), which consist of look-up tables (LUTs) with at most $k$ inputs. Synthesis proceeds in two steps: (i) each $k$-LUT is mapped into a reversible single-target gate with $k$ control lines, (ii) each reversible single-target gate is mapped into a Clifford $+T$ network. LHRS provides different methods to perform the second step. One method, the so-called direct mapping, makes use of the ESOP representation of the control function 
of a reversible single-target gate, which can be directly translated into multiplecontrolled Toffoli gates 6 (see Section 2.2) and further translated into quantum gates 11. The flow of this method is shown in Fig. 2(a).

A second strategy (Fig. 2(b)) for quantum compilation is based on decomposing the initial function, given as a permutation, using the Young-subgroup method described in 3]. It only differs from the first one for the function's specification and the decomposition strategy employed. Differences that will result in a less scalable flow. The final steps are shared between the two flows: ESOP-based reversible synthesis is used to generate a Toffoli network and successively each Toffoli gate is compiled into quantum operations from the Clifford $+T$ library using the method described in [11.

In this work, we address the Clifford $+\mathrm{T}$ universal quantum library, and try to optimize the number of $T$ gates by applying ESOP optimization to the compilation flows. Nevertheless, our analysis and methods are applicable to the other quantum libraries, as far as the implementations of Toffoli gates are known.

\section{Motivation}

In the following, we introduce the problem of finding the right ESOP synthesis method to generate reversible circuits, which can be compiled into quantum circuits with optimal characteristics: minimal number of $T$ gates and reduced number of Clifford gates.

Example 2. Given the Boolean function $f(x)=\bar{x}_{1} \bar{x}_{3} x_{4} \vee \bar{x}_{2} \bar{x}_{3} x_{4} \vee \bar{x}_{1} x_{2} x_{3} \bar{x}_{4} \vee$ $x_{1} \bar{x}_{2} x_{3} \bar{x}_{4}$ with $x=x_{1}, \ldots, x_{4}$, two possible ESOP expressions for $f$ are:

$$
\begin{array}{rr}
A(x)= & x_{3} x_{1} \oplus \bar{x}_{4} x_{1} \oplus x_{3} x_{2} \oplus x_{1} \oplus \bar{x}_{4} x_{2} \oplus x_{2} \oplus x_{4} \bar{x}_{3} \bar{x}_{2} \bar{x}_{1} \\
B(x)= & \bar{x}_{4} x_{3} x_{1} \oplus x_{4} \bar{x}_{3} x_{2} x_{1} \oplus \bar{x}_{4} x_{3} x_{2} \oplus x_{4} \bar{x}_{3}
\end{array}
$$

The first expression $A(x)$ is composed of 7 product terms while the other expression, $B(x)$, is smaller and has size 4 . We can use these ESOPs to synthesize a reversible network for $f$ and successively we can compile them into quantum gates using the algorithm described in [11. The resulting networks and the composition of the quantum circuits are reported in Fig $3 \mathrm{H}$ is the number of Hadamard gates, NOT and CNOT are respectively the number of $\mathrm{X}$ and the number of controlled-X gates, $T$ is the number of $T$ gates. It is clearly shown how the second ESOP, independently from the smaller size, generates a quantum circuit with more gates. Differently, the first ESOP, that has larger size, shows characteristics allowing the compiler to create a circuit with reduced $T$ gates, and fewer gates in general. We want to identify which are the characteristics that lead to a better quantum circuit. With this in mind, we can notice how the first ESOP has cubes with less literals, with respect to the second ESOP. Thus $A(x)$ generates a reversible circuit with multiple-controlled Toffoli gates with less controls and consequently a quantum circuit with less $T$ gates. 

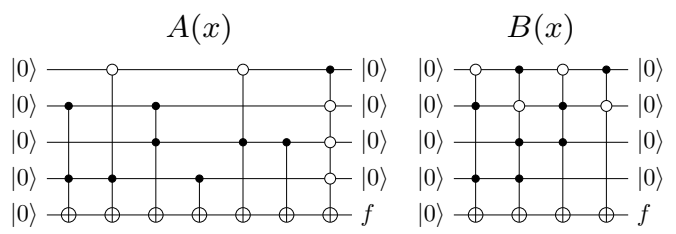

\begin{tabular}{|c|c|c|c|}
\hline \multirow[t]{2}{*}{ ESOP } & \multicolumn{3}{|c|}{ Gate types } \\
\hline & \multicolumn{3}{|c|}{ H NOT CNOT $T$} \\
\hline$A(x)$ & 18 & 6 & 4652 \\
\hline$B(x)$ & 24 & 6 & 5463 \\
\hline
\end{tabular}

Fig. 3. Synthesis results of two different ESOPs for the same function $f$

It is evident how the quantum compilation problem can take advantage of optimal ESOP synthesis strategies. Consequently, in this work we apply stateof-the-art ESOP synthesis and optimization methods, e.g., the heuristic EXORCISM 14, into recent quantum compilation flows. In addition, we present a constraint-based ESOP synthesis method that accepts an arbitrary cost function, as Example2 2 suggests that different cost metrics should be considered for ESOPs in quantum compilation.

\section{Constraint-based ESOP synthesis}

The problem of finding an ESOP expression that realizes a Boolean function is known as ESOP synthesis. The seminal work of Perkowski and ChrzanowskaJeske [17] introduces the Helliwell decision function to characterize the solution space of ESOP synthesis for a given Boolean function.

\subsection{Helliwell decision function}

The Helliwell decision function $H_{f}\left(g_{1}, \ldots, g_{K}\right), K \leq 3^{n}$, for a given Boolean function $f\left(x_{1}, \ldots, x_{n}\right)$ describes synthesis as an odd-even covering problem in terms of the minterms of $f$. For each possible product term in $n$ Boolean variables, a decision variable $g_{i}, 1 \leq i \leq K$, is introduced. The Helliwell decision function is then defined by the logic equation

$$
\bigwedge_{m \in f}\left(\left(\bigoplus_{g \in I(m)} g\right) \oplus f(m) \oplus 1\right),
$$

where $m \in f$ denotes that $m$ is a minterm of $f$ and $I$ maps each minterm to the decision variables $g_{i_{1}}, \ldots, g_{i_{l}}$ whose product terms are covered by $m$.

The logic equation (1) is constructed in such a way that every satisfying assignment $\hat{g}$ for $g=g_{1}, \ldots, g_{K}$ for $H(g)$ directly corresponds to an ESOP expression functionally equivalent to $f$.

Example 3. Given the Boolean function $f\left(x_{1}, x_{2}\right)=x_{1} \vee x_{2}$ with Boolean variables $x_{1}$ and $x_{2}$, the Helliwell decision function using 9 Boolean variables $g_{1}, \ldots, g_{9}$, 

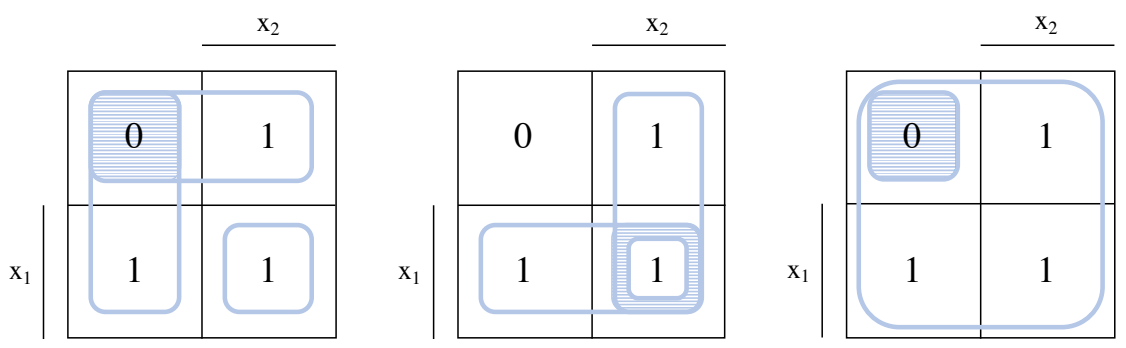

Fig. 4. Three possible ESOP covering for the function $f=x_{1} \vee x_{2}$

that are,

$$
\begin{aligned}
& g_{1}=\bar{x}_{1} \bar{x}_{2} \quad g_{2}=\bar{x}_{1} x_{2} \quad g_{3}=x_{1} \bar{x}_{2} \quad g_{4}=x_{1} x_{2} \\
& g_{5}=x_{1} \quad g_{6}=\bar{x}_{1} \quad g_{7}=x_{2} \quad g_{8}=\bar{x}_{2} \\
& g_{9}=1 .
\end{aligned}
$$

The SAT solver will find a selection of the cubes such that minterms for which $f$ evaluates to one are covered an odd number of times, whether minterms for which $f$ evaluates to false are covered an even number of times. Constraints must be added to the problem in order for the SAT solver to find a valid solution. The overall Helliwell decision function for $f$ is:

$$
\begin{aligned}
H(g)= & \left(g_{1} \oplus g_{6} \oplus g_{8} \oplus g_{9} \oplus 0 \oplus 1\right) \wedge\left(g_{2} \oplus g_{7} \oplus g_{6} \oplus g_{9} \oplus 1 \oplus 1\right) \wedge \\
& \left(g_{3} \oplus g_{5} \oplus g_{8} \oplus g_{9} \oplus 1 \oplus 1\right) \wedge\left(g_{4} \oplus g_{5} \oplus g_{7} \oplus g_{9} \oplus 1 \oplus 1\right)
\end{aligned}
$$

Fig. 4 shows three possible ESOP covers on the Karnaugh map: $g_{4}, g_{6}, g_{8}$ and $g_{4}, g_{5}, g_{7}$ and $g_{6}, g_{9}$.

\subsection{Size-minimal ESOP synthesis}

Size-minimal ESOP synthesis is the problem of finding an ESOP expression for a given Boolean function $f$ with a minimum number of product terms. Utilizing logic equation (1), the problem can be solved by computing minimum satisfying assignments for $H_{f}(g)$. An assignment $\hat{g}$ is minimum satisfying if the two conditions

(a) $H_{f}(\hat{g})$ and (b) $\forall g:\left(g \not \wedge \hat{g} \wedge H_{f}(g)\right) \Longrightarrow g \not \rightarrow \hat{g}$,

hold, i.e., if $\hat{g}$ satisfies $H_{f}$ and no other assignment that satisfies $H_{f}$ implies $\hat{g}$.

In the following, the idea of utilizing the Helliwell decision function for synthesizing size-minimum ESOP expression is generalized to synthesizing costminimal ESOP expressions, where the cost function is provided as a part of the input. 


\subsection{Cost-minimal ESOP synthesis}

Given a Boolean function $f$ over $n$ Boolean variables and a cost function $\kappa$ : $\{0,1,-\}^{n} \rightarrow \mathbb{N}_{>0}$, that maps product terms to positive integer values (costs), cost-minimal ESOP synthesis is the problem of finding an ESOP expression $t_{1} \oplus \cdots \oplus t_{k}$ that realizes $f$ such that $\bigwedge_{i=1}^{k} \kappa\left(t_{i}\right)$ is minimal.

We present two different cost function, $\kappa_{0}$ and $\kappa_{1}$ to illustrate the idea of cost-minimal ESOP synthesis. In general, the cost function should be picked keeping the usage of the ESOP expression in mind.

The constant function

$$
\kappa_{0}(t)=1
$$

defines unit costs for all product terms. If used, each ESOP expression obtained as solution of cost-minimal ESOP synthesis has a minimum number of product terms. The cost function

$$
\kappa_{1}(t)=|t|+1,
$$

where $|t|$ counts the number of literals in $t$, weights each product term by the number of appearing literals. The additional 1 ensures that all costs - including the costs of the empty product term - are greater than 0 .

Example 4. Consider the Boolean function $f_{1}(x)=\bar{x}_{1} \bar{x}_{2} x_{3} x_{4} \vee \bar{x}_{1} x_{2} \bar{x}_{3} x_{4} \vee$ $\bar{x}_{1} x_{2} x_{3} \bar{x}_{4} \vee x_{1} \bar{x}_{2} \bar{x}_{3} x_{4} \vee x_{1} \bar{x}_{2} x_{3} \bar{x}_{4} \vee x_{1} x_{2} \bar{x}_{3} \bar{x}_{4}$ with $x=x_{1}, \ldots, x_{4}$. A cost-minimal ESOP expression that realizes $f_{1}$ with respect to cost function $\kappa_{0}$ is

$$
\bar{x}_{1} x_{2} \bar{x}_{4} \oplus x_{2} \bar{x}_{3} \oplus \bar{x}_{2} x_{3} \bar{x}_{4} \oplus \bar{x}_{1} \bar{x}_{2} x_{3} \oplus x_{1} \bar{x}_{3} x_{4},
$$

whereas a cost-minimal ESOP expression for the same Boolean function with respect to cost functions $\kappa_{1}$ is

$$
x_{1} \oplus x_{2} \oplus \bar{x}_{3} \oplus x_{4} \oplus \bar{x}_{1} \bar{x}_{2} \bar{x}_{3} \bar{x}_{4} \oplus x_{1} x_{2} x_{3} x_{4} .
$$

\subsection{Computing cost-minimal ESOPs}

Next, we present the proposed SAT-based procedure for computing cost-minimal ESOP expressions using (weighted) maximum satisfiability (MAX-SAT) [10.

MAX-SAT deals with solving over-constrained constraint satisfaction problems modulo Boolean logic. The problems consist of hard and soft clauses, where each soft clause is associated with an integer weight greater than 0 . The constraint satisfaction problem initially is unsatisfiable and the task of a MAXSAT oracle is to find a minimal-cost relaxation of the soft clauses, i.e., the oracle has to remove a subset of the soft clauses, such that the problem becomes satisfiable while a given cost function is minimized.

Given a Boolean function $f$ over $n$ Boolean variables and a cost function $\kappa$ : $\{0,1,-\}^{n} \rightarrow \mathbb{N}_{>0}$, cost-minimal ESOP synthesis is solved in three steps: 


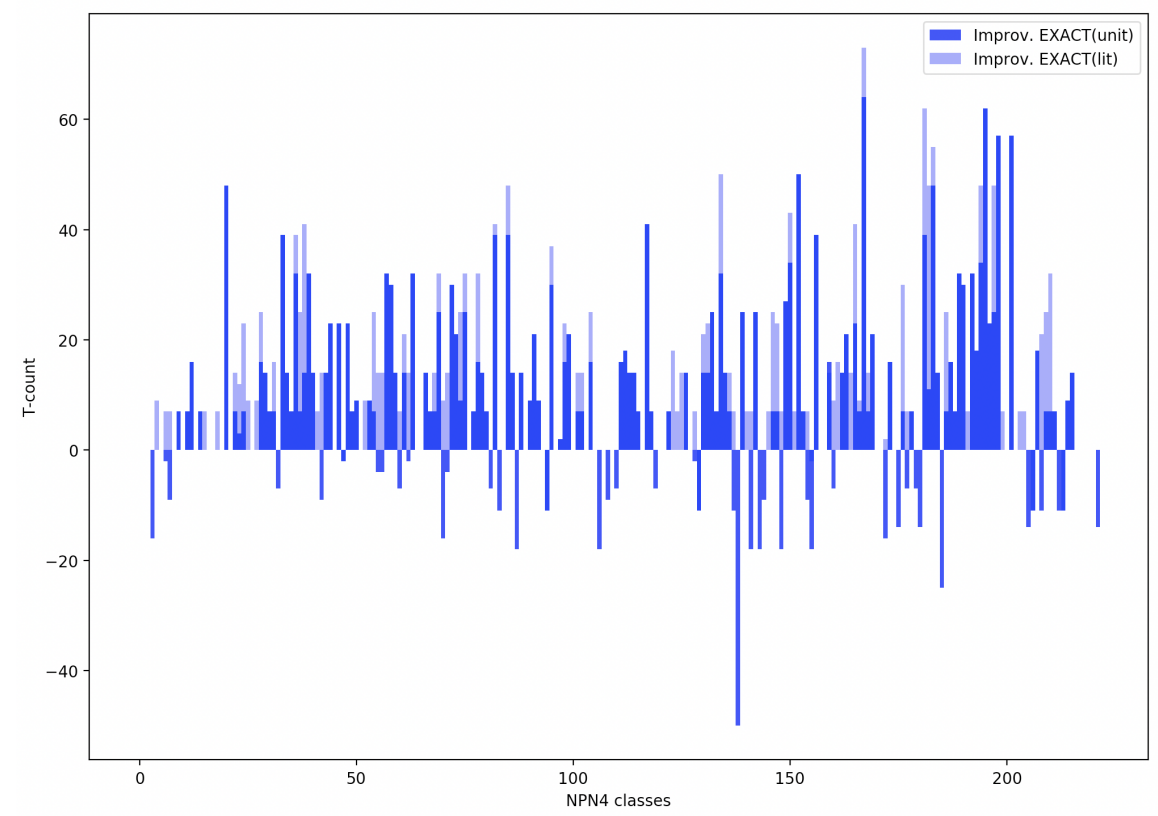

Fig. 5. Histogram showing the improvement over exact methods over $P K R M$ with respect to two different cost functions: number of terms (EXACT(unit)) and number of literals $(E X A C T($ lit $))$

1. Formulate the Helliwell decision function $H(g)$ as described in (1).

2. Invoke a MAX-SAT oracle to find a satisfying assignment $\hat{g}=\hat{g}_{1}, \ldots, \hat{g}_{K}$ that minimizes $\sum_{i=1}^{K} \kappa\left(g_{i}\right)$ subject to $\mathrm{CNF}[H(g)] \wedge\left(\bigwedge_{i=1}^{n} \bar{g}_{i}\right)$, where $\mathrm{CNF}$ translates the XOR-clauses to conjunctive normal form (CNF).

3. Construct the ESOP from the satisfying assignment $\hat{g}$.

The described approach is independent of the choice of the MAX-SAT oracle and the translation to CNF, but uses them as black-boxes.

\section{Results}

\subsection{NPN4 equivalence classes}

In this section, we evaluate the effect of different ESOP optimization methods on simple Boolean functions. As benchmarks, we use the 222 representatives of the NPN4 equivalence classes. We evaluate the number of product terms in the ESOP, as well as, the number of $T$ gates in the generated quantum circuits considering different ESOP synthesis methods and the proposed constraint-based approach: 


\begin{tabular}{lrrrrr}
\hline Cost function & \multicolumn{4}{c}{ ESOP Synthesis Method } \\
\cline { 2 - 6 } & PPRM & PKRM EXORCISM EXACT(unit) & EXACT(lit) \\
\hline avg. ESOP size & 7.77 & 4.69 & 3.41 & 3.41 & 3.42 \\
avg. num. $T$ gates & 87.35 & 82.32 & 59.05 & 67.50 & 58.19 \\
\hline
\end{tabular}

Table 1. Comparison of different ESOP synthesis methods

1. Positive Polarity Reed Muller (PPRM) 29,

2. Pseudo-Kronecker Reed Muller (PKRM) 4,

3. EXORCISM 14 and

4. EXACT(unit) and EXACT(lit) minimizing respectively $\kappa_{0}$ and $\kappa_{1}$

We report the average number of product terms (size) and the average number of $T$ gates for each of the ESOP synthesis methods in Table 1 PPRM and $P K R M$ are special cases of general ESOP expressions, that can be easily derived from a given Boolean function but are sub-optimal when considering the number of product terms. They are often used as starting covers for ESOP optimization approaches. We report them to enable better comparability of the achieved reduction. EXORCISM is a fast cube transformation heuristic, capable of finding close to optimal ESOP expressions, starting from a PKRM cover of the Boolean function. Nevertheless, EXORCISM is an heuristic method and does not guarantee the minimality of the solution. In many cases, reducing the size of an ESOP also leads to a reduction of the number of $T$ gates. Consequently, EXORCISM, EXACT(unit), and EXACT(lit) improve over PPRM and PRKM. Reducing the number of literals also has a positive effect on the $T$ gates, i.e., EXACT(lit) achieves a better reduction than EXACT(unit). Moreover, EXOR$C I S M$ also improves over the EXACT(unit) method because its heuristic prefers don't cares over concrete values and reduces the overall number of literals in an ESOP expression.

The histogram in Fig. 5 gives a more detailed overview of the improvement in T-count of EXACT(lit) and EXACT(unit) over PKRM, respectively, for all the 222 representatives in NPN4 equivalent classes.

Optimizing size and literals, however, does not minimize the number of $T$ gates, which we illustrate by example: consider the two equivalent ESOPs

$$
C\left(x_{1}, x_{2}, x_{3}\right)=1 \oplus \bar{x}_{1} x_{2} \oplus x_{1} x_{2} x_{3} \quad \text { and } D\left(x_{1}, x_{2}, x_{3}\right)=x_{1} x_{2} \bar{x}_{3} \oplus \bar{x}_{2} \oplus \bar{x}_{1} .
$$

Both ESOPs have the same number of product terms and the same number of literals. To realize $C\left(x_{1}, x_{2}, x_{3}\right)$ as quantum circuit, however, $23 T$ gates are required, whereas for realizing $D\left(x_{1}, x_{2}, x_{3}\right) 16 T$ gates are needed. This results suggest that in future work it would be valuable to identify more fitting cost functions than the number of literals. In addition, future technology developments could themselves require different cost functions. Our proposed constraint-based method could provide the flexibility to enable future research in this direction. 


\subsection{Integration into quantum compilation flows}

In this section, we show the result of integrating the advanced ESOP optimization methods into the quantum compilation flows $D B S$ and $L H R S$.

To integrate optimized ESOP synthesis methods, we propose a pseudo-optimal portfolio approach as described in Alg. 1. For each symmetric control function, the ESOP expression esop is computed using the PKRM method, that is optimum in this case. If the number of inputs is smaller or equal to 4 , we use the exact methods to minimize the number of literals. For larger functions the heuristic EXORCISM is used (command \&exorcism - $q$ of abc [2]).

First we evaluate the improvement of the proposed method integrated into $D B S$ (Fig. 2(b)). In Table 2 we show the synthesis results for reversible permutations from Maslov's reversible benchmark ${ }^{1}$. In addition we created reversible functions $M O D_{n / g}: \mathbb{B}^{n} \rightarrow \mathbb{B}^{n}$, where:

$$
M O D_{n / g}= \begin{cases}0 & \text { if } \quad x=0 \\ g^{x} \bmod \left(2^{n}-1\right) & \text { if } \quad 1 \leq x \leq 2^{n}-2 \\ 2^{n}-1 & \text { otherwise }\end{cases}
$$

The data are showing a reduction in the number of $T$ gates, with respect to the PKRM method, for both the EXACT approaches. Nevertheless, we can see how, if the synthesis is performed to minimize the number of literals in each cube, the $T$-count can be further improved. In fact, the unit approach gets to $22.66 \%$ improvement, while lit gives $28.23 \%$ improvement.

In a second experiment, we evaluate the integration into the LHRS framework. In Table 3 we show results of synthesizing the arithmetic designs of the EPFL benchmark ${ }^{2}$ into quantum circuits. As explained in the preliminary section, the first steps of the flow generate a reversible circuit made of singletarget gates, each one with a control function of maximum $k$ inputs, where $k$ is the LUT size used to build the k-LUT network. An ESOP expression is synthesized for each control function and translated into quantum circuits as described in [6, 11]. We compare a flow integrating our pseudo-exact approach against a flow using PKRM for the mapping of single-target gates. We report synthesis results for LUT size $(k)$ from 4 to 10 . We obtain a maximum reduction of number of $T$ gates in the case of $k=10$ equal to $36.32 \%$ and a minimum reduction in the case of $k=4$ equal to $17.86 \%$.

\section{Open source implementation}

The proposed SAT-based exact synthesis method is implemented in the open source C++ library easy ${ }^{3}$ 19, 24 using our own $\mathrm{C}++$ implementation of RC2 8 as MAX-SAT oracle. The easy library provides implementations of various verification and synthesis algorithms for ESOP expressions.

\footnotetext{
1 http://webhome.cs.uvic.ca/ dmaslov

2 https://github.com/lsils/benchmarks

3 https://github.com/hriener/easy
} 


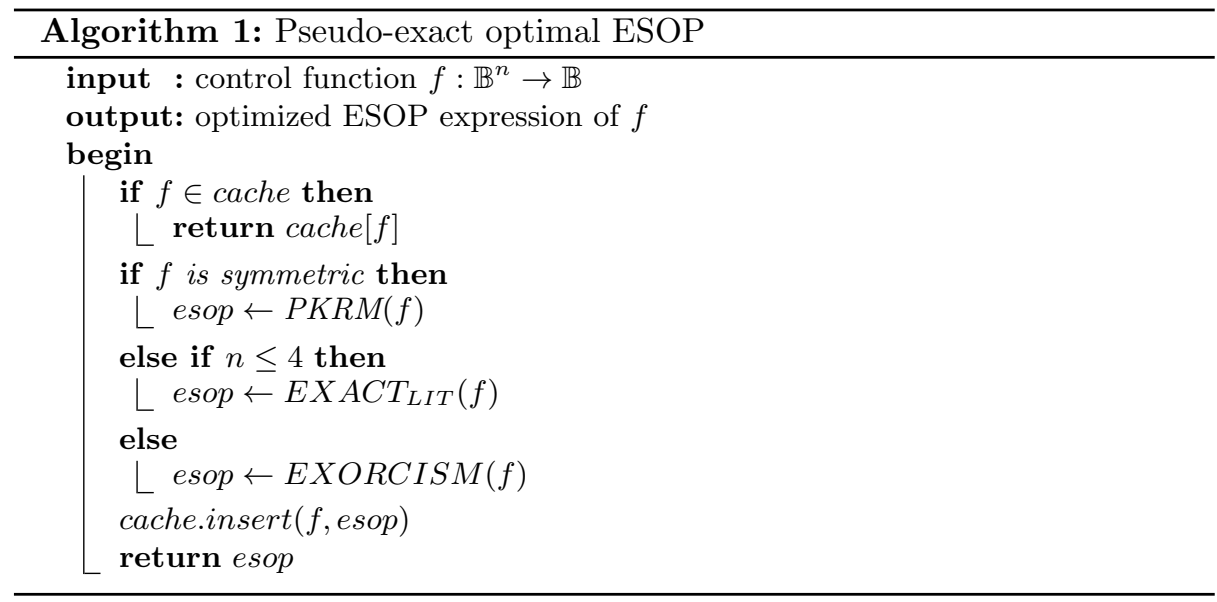

Table 2. Comparison between exact method and heuristic for small reversible functions

\begin{tabular}{|c|c|c|c|c|c|c|c|}
\hline \multirow[t]{2}{*}{ Permutation } & \multirow[b]{2}{*}{ Q } & \multicolumn{2}{|c|}{ PKRM } & \multicolumn{2}{|c|}{ EXACT(lit) } & \multicolumn{2}{|c|}{ EXACT(unit) } \\
\hline & & $\mathrm{T}$ & $\mathrm{t}[\mathrm{s}]$ & $\mathrm{T}$ & $\mathrm{t}[\mathrm{s}]$ & $\mathrm{T}$ & $\mathrm{t}[\mathrm{s}]$ \\
\hline hwb4 & 4 & 123 & 0.0 & 109 & 0.1 & 116 & 0.0 \\
\hline hwb5 & 5 & 514 & 0.0 & 337 & 59.9 & 447 & 0.3 \\
\hline hwb6 & 6 & 1361 & 0.0 & 993 & 0.9 & 993 & 0.9 \\
\hline hwb7 & 7 & 5331 & 0.0 & 3066 & 1.0 & 3066 & 1.1 \\
\hline hwb8 & 8 & 13562 & 0.0 & 7654 & 1.2 & 7654 & 1.2 \\
\hline $\bmod 5 \_11$ & 5 & 453 & 0.0 & 350 & 36.5 & 368 & 0.2 \\
\hline $\bmod 5 \_12$ & 5 & 453 & 0.0 & 361 & 59.1 & 400 & 0.2 \\
\hline $\bmod 5 \_13$ & 5 & 428 & 0.0 & 329 & 38.2 & 343 & 0.1 \\
\hline $\bmod 5 \_17$ & 5 & 478 & 0.0 & 382 & 64.3 & 414 & 0.3 \\
\hline $\bmod 5 \_21$ & 5 & 433 & 0.0 & 352 & 34.9 & 482 & 0.1 \\
\hline $\bmod 5 \_22$ & 5 & 469 & 0.0 & 354 & 25.0 & 391 & 0.1 \\
\hline $\bmod 5 \_24$ & 5 & 503 & 0.0 & 405 & 61.4 & 448 & 0.3 \\
\hline $\bmod 5 \_3$ & 5 & 494 & 0.0 & 386 & 34.8 & 411 & 0.2 \\
\hline $\bmod 7 \_14$ & 7 & 5201 & 0.0 & 2936 & 1.0 & 2936 & 1.0 \\
\hline $\bmod 7 \_3$ & 7 & 4945 & 0.0 & 2957 & 1.0 & 2957 & 1.0 \\
\hline $\bmod 7-7$ & 7 & 4859 & 0.0 & 3039 & 1.0 & 3039 & 1.0 \\
\hline prime4 & 4 & 102 & 0.0 & 95 & 0.0 & 106 & 0.0 \\
\hline prime5 & 5 & 367 & 0.0 & 271 & 28.5 & 289 & 0.1 \\
\hline prime6 & 6 & 1054 & 0.0 & 786 & 0.8 & 786 & 0.7 \\
\hline prime 7 & 7 & 3600 & 0.0 & 2283 & 1.0 & 2283 & 0.9 \\
\hline prime8 & 8 & 8302 & 0.0 & 4420 & 1.1 & 4420 & 1.0 \\
\hline
\end{tabular}

For the quantum compilation results, we interfaced easy with caterpillar $^{4}$ and tweedledum ${ }^{5}$. The first library is dedicated to quantum compilation, hierarchical methods, and quantum memory management, whereas the second library implements state-of-the-art synthesis methods, e.g., Young subgroup decomposition based synthesis.

\footnotetext{
4 https://github.com/gmeuli/caterpillar

5 https://github.com/boschmitt/tweedledum
} 
Table 3. Synthesis of EPFL arithmetic benchmark on

\begin{tabular}{|c|c|c|c|c|c|c|c|c|c|c|c|c|}
\hline \multirow[b]{2}{*}{$\mathrm{k}$} & & \multirow[b]{2}{*}{ Q } & \multicolumn{2}{|c|}{ PKRM } & \multicolumn{2}{|c|}{ Opt. } & & \multirow[b]{2}{*}{$\mathrm{Q}$} & \multicolumn{2}{|c|}{ PKRM } & \multicolumn{2}{|c|}{ Opt. } \\
\hline & & & $\mathrm{T}$ & $\mathrm{t}[\mathrm{s}]$ & $\mathrm{T}$ & $\mathrm{t}[\mathrm{s}]$ & & & $\mathrm{T}$ & $\mathrm{t}[\mathrm{s}]$ & $\mathrm{T}$ & $\mathrm{t}[\mathrm{s}]$ \\
\hline 4 & adder & 511 & 5398 & 0.0 & 5356 & 0.4 & bar & 1415 & 76816 & 0.2 & 56320 & 1.8 \\
\hline 5 & & 448 & 16061 & 0.1 & 15151 & 0.5 & & 1031 & 95576 & 0.3 & 63694 & 2.9 \\
\hline 6 & & 448 & 16271 & 0.1 & 15279 & 0.6 & & 647 & 52750 & 0.2 & 50944 & 1.8 \\
\hline 7 & & 427 & 37259 & 0.1 & 36110 & 0.7 & & 647 & 52750 & 0.3 & 50944 & 1.9 \\
\hline 8 & & 427 & 37963 & 0.1 & 36654 & 0.7 & & 647 & 52750 & 0.3 & 50944 & 1.9 \\
\hline 9 & & 416 & 84076 & 0.2 & 72338 & 0.8 & & 647 & 52750 & 0.3 & 50944 & 1.9 \\
\hline 10 & & 416 & 85509 & 0.2 & 72985 & 0.9 & & 647 & 52750 & 0.3 & 50944 & 1.9 \\
\hline 4 & div & 26467 & 757193 & 5.8 & 635999 & 12.4 & hyp & 64630 & 2448872 & 25.3 & 2208000 & 37.5 \\
\hline 5 & & 24474 & 851035 & 6.8 & 690622 & 15.1 & & 56568 & 2647894 & 26.1 & 2156087 & 40.5 \\
\hline 6 & & 24083 & 876636 & 8.0 & 709586 & 19.0 & & 50118 & 2860466 & 28.2 & 2145634 & 46.6 \\
\hline 7 & & 23944 & 939887 & 9.6 & 742327 & 23.8 & & 48399 & 3501767 & 31.0 & 2817812 & 51.8 \\
\hline 8 & & 23808 & 1034583 & 11.2 & 773058 & 26.6 & & 47581 & 4540244 & 36.9 & 3546120 & 66.7 \\
\hline 9 & & 23711 & 1204407 & 13.0 & 831482 & 30.5 & & 46992 & 5379295 & 43.0 & 4158260 & 79.1 \\
\hline 10 & & 23633 & 1710038 & 15.4 & 875766 & 34.3 & & 46933 & 6238649 & 50.0 & 4596940 & 94.4 \\
\hline 4 & $\log$ & 10420 & 458335 & 2.4 & 380787 & 12.8 & $\max$ & 1484 & 54422 & 0.2 & 42684 & 5.4 \\
\hline 5 & & 9661 & 623957 & 3.2 & 492501 & 24.1 & & 1346 & 76507 & 0.2 & 60597 & 6.4 \\
\hline 6 & & 8156 & 1033225 & 4.3 & 768429 & 49.4 & & 1256 & 104109 & 0.3 & 79853 & 6.4 \\
\hline 7 & & 8141 & 1507690 & 5.1 & 883462 & 103.7 & & 1149 & 148355 & 0.4 & 102310 & 6.0 \\
\hline 8 & & 4658 & 2196359 & 6.2 & 1228593 & 48.1 & & 1067 & 209851 & 0.6 & 140106 & 6.9 \\
\hline 9 & & 4456 & 3393095 & 8.4 & 1912337 & 65.8 & & 977 & 323027 & 0.8 & 200270 & 5.9 \\
\hline 10 & & 3697 & 5786642 & 10.8 & 3268408 & 74.8 & & 929 & 355341 & 1.1 & 230118 & 5.6 \\
\hline 4 & mult & 8194 & 359422 & 1.8 & 270268 & 6.2 & $\sin$ & 1962 & 71409 & 0.4 & 64103 & 14.5 \\
\hline 5 & & 8100 & 479930 & 2.2 & 368062 & 8.8 & & 1818 & 82386 & 0.5 & 71471 & 19.4 \\
\hline 6 & & 6706 & 1034190 & 2.8 & 579420 & 11.6 & & 1608 & 115107 & 0.7 & 92659 & 25.7 \\
\hline 7 & & 7050 & 1448336 & 3.7 & 847558 & 15.2 & & 1553 & 137989 & 0.9 & 104092 & 27.3 \\
\hline 8 & & 5101 & 1371054 & 3.7 & 818914 & 16.6 & & 1449 & 249964 & 1.2 & 157332 & 32.5 \\
\hline 9 & & 5165 & 2115333 & 5.2 & 1410009 & 18.3 & & 915 & 794521 & 1.5 & 362082 & 33.2 \\
\hline 10 & & 4006 & 3657831 & 8.0 & 2417393 & 23.9 & & 878 & 1241237 & 2.2 & 542136 & 37.2 \\
\hline 4 & sqrt & 8686 & 317522 & 1.7 & 255275 & 6.4 & & 6909 & 354552 & 1.5 & 240636 & 11.5 \\
\hline 5 & & 8351 & 344049 & 2.3 & 265948 & 6.8 & & 6092 & 553311 & 1.9 & 308262 & 18.0 \\
\hline 6 & & 8332 & 391900 & 2.9 & 285310 & 7.8 & & 4195 & 299574 & 1.8 & 206683 & 16.1 \\
\hline 7 & & 8152 & 448518 & 3.4 & 301246 & 8.4 & & 4213 & 368160 & 2.0 & 261272 & 22.8 \\
\hline 8 & & 7986 & 709282 & 4.4 & 358215 & 9.5 & & 3764 & 477446 & 2.3 & 341092 & 24.3 \\
\hline 9 & & 7976 & 720144 & 5.3 & 359296 & 10.6 & & 3724 & 658343 & 2.9 & 445505 & 32.5 \\
\hline 10 & & 7966 & 1413589 & 7.0 & 540586 & 12.4 & & 3792 & 876884 & 3.7 & 532124 & 41.4 \\
\hline
\end{tabular}

min avg. improvement $\mathrm{k}=4: 17.86 \%$

$\max$ avg. improvement $\mathrm{k}=10: 36.32 \%$

avg. improvement: $26.36 \%$

\section{Conclusion}

In this work, we integrate ESOP synthesis methods into quantum compilation flows in order to improve the quality of the produced quantum circuits. We target fault-tolerant quantum computing and aim at minimizing the number of expensive $T$ gates. We consider two different compilation flows for Boolean functions that make use of ESOP-based reversible synthesis.

For both frameworks this integration leads to promising results, which show maximum $T$-count reductions of $28.23 \%$ in $D B S$ and $36.32 \%$ in $L H R S$ with respect to $P K R M$. In conclusion, advanced ESOP synthesis methods, both exact and heuristic, can be applied inside the quantum compilation flows that use 
ESOP-based reversible synthesis, to generate better circuits for fault-tolerant quantum computing.

Acknowledgments This research was supported by the European COST Action IC 1405 'Reversible Computation', by the EPFL Open Science Fund and the Institutional Strategy of the University of Bremen, funded by the German Excellence Initiative.

\section{References}

1. Amy, M., Maslov, D., Mosca, M., Roetteler, M.: A meet-in-the-middle algorithm for fast synthesis of depth-optimal quantum circuits. IEEE Transactions on ComputerAided Design of Integrated Circuits and Systems 32(6), 818-830 (2013)

2. Brayton, R., Mishchenko, A.: ABC: An academic industrial-strength verification tool. In: International Conference on Computer Aided Verification. pp. 24-40. Springer (2010)

3. De Vos, A., Van Rentergem, Y.: Young subgroups for reversible computers. Advances in Mathematics of Communications 2(2), 183-200 (2008)

4. Drechsler, R.: Pseudo-kronecker expressions for symmetric functions. IEEE Transactions on Computers 48(9), 987-990 (1999)

5. Drechsler, R., Finder, A., Wille, R.: Improving ESOP-based synthesis of reversible logic using evolutionary algorithms. In: European Conference on the Applications of Evolutionary Computation. pp. 151-161. Springer (2011)

6. Fazel, K., Thornton, M., Rice, J.E.: ESOP-based Toffoli gate cascade generation. In: IEEE Pacific Rim Conference on Communications, Computers and Signal Processing. pp. 206-209 (2007)

7. Haener, T., Soeken, M., Roetteler, M., Svore, K.M.: Quantum circuits for floatingpoint arithmetic. In: International Conference on Reversible Computation. pp. 162174. Springer (2018)

8. Ignatiev, A., Morgado, A., Marques-Silva, J.: PySAT: A Python toolkit for prototyping with SAT oracles. In: Theory and Applications of Satisfiability Testing. pp. $428-437$ (2018)

9. JavadiAbhari, A., Patil, S., Kudrow, D., Heckey, J., Lvov, A., Chong, F.T., Martonosi, M.: ScaffCC: A framework for compilation and analysis of quantum computing programs. In: Proceedings of the 11th ACM Conference on Computing Frontiers. p. 1. ACM (2014)

10. Li, C.M., Manyà, F.: MaxSAT, hard and soft constraints. In: Handbook of Satisfiability, pp. 613-631 (2009)

11. Maslov, D.: Advantages of using relative-phase Toffoli gates with an application to multiple control Toffoli optimization. Physical Review A 93(2), 022311 (2016)

12. Miller, D.M., Wille, R., Drechsler, R.: Reducing reversible circuit cost by adding lines. In: 2010 40th IEEE International Symposium on Multiple-Valued Logic. pp. 217-222. IEEE (2010)

13. Mishchenko, A., Chatterjee, S., Brayton, R.K.: Improvements to technology mapping for LUT-based FPGAs. IEEE Transactions on Computer-Aided Design of Integrated Circuits and Systems 26(2), 240-253 (2007)

14. Mishchenko, A., Perkowski, M.: Fast heuristic minimization of exclusive-sums-ofproducts. Proc. Int. Workshop Appl. ReedMuller Expansion Circuit Des. pp. 242$250(2001)$ 
15. Mizuki, T., Otagiri, T., Sone, H.: An application of ESOP expressions to secure computations. Journal of Circuits, Systems, and Computers 16(02), 191-198 (2007)

16. Papakonstantinou, K., Papakonstantinou, G.: A nonlinear integer programming approach for the minimization of boolean expressions. Journal of Circuits, Systems and Computers 27(10), 1850163 (2018)

17. Perkowski, M., Chrzanowska-Jeske, M.: An exact algorithm to minimize mixedradix exclusive sums of products for incompletely specified Boolean functions. In: ISCAS. pp. 1652-1655 (1990)

18. Rawski, M.: Application of functional decomposition in synthesis of reversible circuits. In: International Conference on Reversible Computation. pp. 285-290. Springer (2015)

19. Riener, H., Ehlers, R., Schmitt, B., De Micheli, G.: Exact synthesis of ESOP forms. CoRR abs/1807.11103 (2018), http://arxiv.org/abs/1807.11103

20. Sasao, T.: EXMIN2: A simplification algorithm for exclusive-or-sum-of-products expressions for multiple-valued-input two-valued-output functions. IEEE Transactions on Computer-Aided Design of Integrated Circuits and Systems 12(5), 621632 (1993)

21. Sasao, T.: Representations of logic functions using EXOR operators. In: Representations of discrete functions, pp. 29-54. Springer (1996)

22. Soeken, M., Haener, T., Roetteler, M.: Programming quantum computers using design automation. In: Design, Automation \& Test in Europe Conference \& Exhibition (DATE), 2018. pp. 137-146. IEEE (2018)

23. Soeken, M., Mozafari, F., Schmitt, B., De Micheli, G.: Compiling permutations for superconducting QPUs. In: DATE. p. To appear (2019)

24. Soeken, M., Riener, H., Haaswijk, W., Micheli, G.D.: The EPFL logic synthesis libraries. CoRR abs/1805.05121 (2018), http://arxiv.org/abs/1805.05121

25. Soeken, M., Roetteler, M., Wiebe, N., De Micheli, G.: Logic synthesis for quantum computing. CoRR abs/1706.02721 (2017), http://arxiv.org/abs/1706.02721

26. Soeken, M., Roetteler, M., Wiebe, N., De Micheli, G.: LUT-based hierarchical reversible logic synthesis. IEEE Transactions on Computer-Aided Design of Integrated Circuits and Systems (2018)

27. Stergiou, S., Daskalakis, K., Papakonstantinou, G.: A fast and efficient heuristic ESOP minimization algorithm. In: Proceedings of the 14th ACM Great Lakes symposium on VLSI. pp. 78-81. ACM (2004)

28. Wille, R., Soeken, M., Otterstedt, C., Drechsler, R.: Improving the mapping of reversible circuits to quantum circuits using multiple target lines. In: 2013 18th Asia and South Pacific Design Automation Conference (ASP-DAC). pp. 145-150. IEEE (2013)

29. Zhegalkin, I.: The technique of calculation of statementsin symbolic logic. In: Mathe. Sbornik. vol. 34, pp. 9-28 (1927), (in Russian) 